\title{
PREDICTORS OF ENUCLEATION AND MORCELLATION TIME DURING HOLMIUM LASER ENUCLEATION OF THE PROSTATE
}

M Francesca Monn, Marawan El Tayeb, Naeem Bhojani, Matthew J. Mellon, James C. Sloan, Ronald S. Boris, James E. Lingeman

Indiana University School of Medicine, Department of Urology, Indianapolis, IN

\section{Address all correspondence to:}

James E. Lingeman, M.D.

Indiana University School of Medicine

1801 North Senate Blvd. Suite 220

Indianapolis, IN 46202

Tel: 317.962 .2485

Fax: 317.962.2893

E-mail: jlingeman@iuhealth.org

\section{Word Count:}

Abstract: 208

Text: 2060

Running head: Predictors of HoLEP time

Key Words: HoLEP, enucleation, morcellation, BPH

This is the author's manuscript of the article published in final edited form as:

Monn, M. F., El Tayeb, M., Bhojani, N., Mellon, M. J., Sloan, J. C., Boris, R. S., \& Lingeman, J. E. (2015).

Predictors of Enucleation and Morcellation Time During Holmium Laser Enucleation of the Prostate. Urology, 86(2), 338-342. http://doi.org/10.1016/j.urology.2015.04.028 


\section{Abstract}

Objectives: To examine predictors of enucleation and morcellation time within a large cohort of men undergoing HoLEP for benign prostatic hypertrophy.

Methods: Pre-operative, peri-operative, and post-operative clinical characteristics were available from men treated with HoLEP between 1998 and 2013 at Indiana University Health Methodist Hospital. Stepwise linear regression was performed to determine clinical variables which are associated with enucleation and morcellation times.

Results: We identified 960 patients who underwent HoLEP. Average (range) enucleation time was 65.7 (11-245) minutes and morcellation time was 19.7 (3-260) minutes. History of anticoagulation was associated with a small decrease in enucleation time $(\mathrm{p}=0.013)$ while increasing HoLEP specimen weight was associated with increasing enucleation time $(\mathrm{p}<0.001)$. History of intermittent catheterization, urinary tract infections, presence of dense prostatic tissue (colloquially referred to as "beach balls"), and increasing specimen weight were associated with increasing morcellation time ( $<0.05$ each). Having HoLEP performed by a less experienced urologist was associated with longer enucleation and morcellation times.

Conclusions: Prostate volume is significantly associated with increased enucleation and morcellation times during HoLEP. Additionally, history of UTI and CIC are associated with modest increases in enucleation and morcellation times. Dense enucleated prostate tissue significantly impacts the ability to morcellate effectively. Increasing surgeon experience can significantly improve both enucleation and morcellation efficiency. 


\section{Introduction}

Since its introduction in the late $1990 \mathrm{~s}^{1}$, holmium laser enucleation of the prostate (HoLEP) has increased in popularity for management of benign prostatic hyperplasia (BPH). Evidence suggests that HoLEP provides superior outcomes compared to TURP and open simple prostatectomy in men with $\mathrm{BPH}$, regardless of prostate size $\mathrm{e}^{2-5}$. Additionally, a recent study found that HoLEP is associated with decreased hospital costs compared to open prostatectomy. ${ }^{6}$

Performance and efficiency of HoLEP relies on two critical steps-transitional zone enucleation followed by tissue morcellation. Enucleation and morcellation times are dependent upon a number of factors including prostatic volume, as well as tissue quality. Because a steep learning curve and the potential for long operative times with HoLEP are common criticisms of the procedure, we sought to evaluate pre-HoLEP variables that may impact both enucleation and morcellation times during surgery.

\section{Materials and methods}

Patients, outcomes, and variables

After obtaining Institutional Review Board (IRB) approval (Protocol \#1010002242), we conducted a retrospective cohort analysis using the prospectively collected IU Health Methodist Hospital database to identify patients who underwent HoLEP between 1998 and 2013. The primary outcomes of interest were enucleation and morcellation times. Variables included in the analysis were age, pre-HoLEP PSA, history of 5-alpha reductase inhibitor (5ARI) or alpha blocker therapy, history of urinary retention, history of recurrent urinary tract infections (UTI), history of anticoagulation, transrectal ultrasound (TRUS) prostate volume, final pathological HoLEP specimen weight (<30g, 30-100g, 100-150g, and >150g), presence of prostatic 
malignancy in HoLEP specimen, and surgeon experience (experienced vs. less experienced). The majority of surgeries were performed by a single experienced surgeon (JEL) who has performed over 2,000 HoLEP operations at IU Health Methodist Hospital. The remaining HoLEP cases were performed by six surgeons, all with less than 100 procedures on record at our hospital. Patients who did not have a recorded pre-HoLEP PSA, pertinent medical history details (variables included in the regression), pathologic details, and enucleation or morcellation times were excluded $(\mathrm{n}=560)$. There were no statistical differences in pre-operative PSA (when available) between the patients included in and excluded from the study.

\section{Description of procedure}

Using a $100 \mathrm{~W}$ Ho:YAG laser source, configured with a 550-nm end-fire laser fiber, the HoLEP procedure was performed as previously described ${ }^{7}$. A 28 F continuous-flow resectoscope (Karl Storz Endoscopy, Culver City, CA, USA) with a laser bridge housing a 7 F stabilizing catheter (Cook Urologic, Spencer, IN, USA) was used to enucleate the prostate. Normal saline was used as the irrigant in all cases. Briefly, the enucleation requires laser settings of $2 \mathrm{~J}$ and $40-50 \mathrm{~Hz}$ for the lateral lobes, $2 \mathrm{~J}$ and $20 \mathrm{~Hz}$ for the apical dissection, and $2 \mathrm{~J}$ and 20 Hz to divide the apical mucosal bridges.

Tissue morcellation was achieved using a Lumenis VersaCut ${ }^{\mathrm{TM}}$ tissue morcellator (Lumenis Ltd, Yokneam, Israel) introduced through a Storz nephroscope. Dual irrigation is used to keep the bladder full and to improve visualization. Irrigation was placed approximately 24-30 inches above the level of the bladder. Unusually tough prostatic tissue (termed "beach balls") may occasionally be encountered during enucleation which can prolong morcellation time. 
Often these "beach balls" can be refractory to traditional morcellation techniques. When encountered, management options include removing pieces with grasping forceps, exchanging morcellation blades, and lasering into smaller pieces to facilitate engagement into the morcellator. As a last resort, a small cystotomy can be performed to remove residual tissue.

\section{Statistical analyses}

In order to identify potential predictors of enucleation time and morcellation time, separate forward and backward stepwise linear regression models were performed using $\mathrm{p}<0.2$ as our criteria for model inclusion. All variables included in the descriptive analysis were included in the initial stepwise linear regression models and only variables that were identified as significant $(\mathrm{p}<0.2)$ were included in the final presented multiple linear regression models. $A$ priori $\mathrm{p}<0.05$ was set as the threshold for statistical significance for the final multiple linear regression models. All statistical analyses were performed using Stata version 13.0 (Statacorp, College Station, TX).

\section{Results}

Clinical pre-operative, peri-operative, and post-operative characteristics of our patient cohort are shown in Table 1 . There were a total of 960 patients with an average age of 70 years. The majority of patients had a history of either 5ARI and/or alpha blocker therapy (79\%). Twenty-six percent had urinary retention at the time of HoLEP (Table 1). Nearly 90\% of HoLEPs were performed by a single surgeon. Mean enucleation time was 66 minutes while mean morcellation time was 20 minutes. Enucleation rates varied significantly between experienced and less experienced surgeons with enucleation rates of $1.46 \mathrm{~g} / \mathrm{min}(0.9)$ for 
experienced and $0.77 \mathrm{~g} / \mathrm{min}(0.6)$ for less experienced surgeons $(\mathrm{p}<0.001)$. Similar findings were observed for morcellation rate with $5.45 \mathrm{~g} / \mathrm{min}$ for experienced and $4.21 \mathrm{~g} / \mathrm{min}$ for less experienced surgeons $(\mathrm{p}<0.001)$. Of note, among cases where prostate "beach balls" were mentioned in the operative report ( $\mathrm{n}=34,3.5 \%$ ), the average morcellation time was 51.4 minutes compared with 18.5 minutes in non-“beach ball” patients $(\mathrm{p}<0.001)$.

\section{Enucleation time}

Variables identified from the stepwise linear regression models as being associated with enucleation time included urinary retention, history of UTI, history of anticoagulation, HoLEP specimen weight, presence of "beach balls" in operative report, and surgeon experience (Table

2). In the final model, history of anticoagulation was associated with a five minute decrease in enucleation time ( $\mathrm{p}=0.024)$. Less experienced surgeons had an estimated 21 minute increase in enucleation time $(\mathrm{p}<0.001)$. Compared with specimen weight of $<30$ g, each progressive specimen weight category was associated with significant increases in enucleation time ranging from 18 to 40 minutes (Table 2).

\section{Morcellation time}

Variables identified from the stepwise linear regression models as being associated with morcellation time included history of 5ARI therapy, history of UTI, HoLEP specimen weight, presence of "beach balls", and experience of the surgeon (Table 3). In the final multiple linear regression model, history of UTI was associated with a significant increase in morcellation time by 2.5 minutes. Adjusting for the other covariates in the model, presence of "beach balls" 
conferred a 25 minute increase in morcellation time. Additionally, increasing HoLEP specimen weight conferred a 9-40 minute increase in morcellation time (Table 3).

\section{Discussion}

Considerable level one evidence has demonstrated the benefits of HoLEP over simple prostatectomy and TURP for the treatment of BPH in large glands ${ }^{8-14}$. Amongst established benefits of HoLEP are its long term, durable, and reproducible results that can be applied to any prostate gland size $12,15,16$. A frequently reported criticism of HoLEP is the length of the procedure, as well as the steep learning curve associated with surgical efficiency. Thus, understanding patient and tissue variables that may impact operative time may assist in patient counseling, scheduling of efficient OR time, and proper patient selection for surgeons in various stages of experience.

Enucleation involves complete removal of the transitional zone of the prostate using a holmium-YAG laser. This is not a vaporization procedure but rather the laser is used as a cutting tool. Enucleation time largely depends upon prostate size, configuration, and visualization. Similarly, morcellation time is impacted both by enucleation volume and visualization. As such, it is not surprising that HoLEP specimen weight was associated with significant increases in enucleation and morcellation time. Multiple previous studies have suggested this relationship with many further reporting that HoLEP operative efficiency increases with larger prostate volumes ${ }^{17-20}$. Interestingly, and in line with our findings, studies have suggested that as surgeons become more comfortable with performing HoLEP, the enucleation and morcellation time decreases ${ }^{19}$. Not unexpectedly, patients with "beach ball” enucleated tissue encountered during HoLEP had significantly longer morcellation times. Identifying factors contributing to the 
formation of these dense tissue pieces is worth investigation in future studies. Recently, the introduction of new morcellators (Wolf Piranha, Knittlingen, Germany) has greatly reduced this problem.

Elzayat et al examined the safety of HoLEP in chronically anticoagulated patients and found that HoLEP was a safe option for this patient population ${ }^{21}$. This is not surprising as the holmium-YAG laser effectively coagulates while enucleating the tissue ${ }^{8}$. We initially hypothesized that chronic anti-coagulation may worsen enucleation and/or morcellation times as this could negatively impact visualization during surgery. ${ }^{21}$ Interestingly, our study found that a history of chronic anticoagulation was associated with a small but significant decrease in enucleation time. Although we are unable to explain this relationship, we speculate, because of anticipated concern regarding intra-operative bleeding, that this may actually reflect earlier participation by senior staff and thus decreased resident or fellow participation in the enucleation portion of the case.

The learning curve associated with HoLEP is often considered a disadvantage to the procedure despite studies suggesting that proficiency can be gained in as little as two dozen procedures $^{22,23}$. Furthermore, studies have reported that there is little variation in operative efficiency between surgeons and that comparable times can be achieved ${ }^{17,24}$. We found that although there is a twenty minute increase in enucleation time associated with less experienced surgeons, there is minimal difference in morcellation time. Enucleation is the more timeconsuming portion of HoLEP and we believe that efficiency likely improves with surgeon experience. Of note, the impact of tissue quality, i.e. prostatic "beach balls", is an important determinant of time regardless of surgeon experience, although surgeon experience certainly diminishes the impact that difficult tissue has on overall operative times. 
We examined whether recurrent UTI, chronic anticoagulation, and urinary retention (catheter or CIC leading up to surgery) would impact morcellation or enucleation time. We hypothesized that each of these might play a significant role because of their potential to impact prostatic tissue quality. Recent studies have evaluated the relationship between chronic prostatic inflammation with urinary retention and $\mathrm{BPH}$ progression with the finding that patients with chronic prostate inflammation have significantly higher risk of retention ${ }^{25}$. Recurrent UTI and chronic urinary catheterization can all hypothetically increase prostate inflammation, although studies examining this relationship have failed to demonstrate consistent findings ${ }^{25,26}$. Prostate inflammation impacts the histologic architecture, increasing gland volume and obliterates the natural plane between adenoma and prostate capsule which may result in a more complicated dissection during enucleation. Inflamed prostate tissue may also trigger increased bleeding or oozing while operating which may result in poorer visualization requiring increased time to achieve appropriate hemostasis during surgery. Furthermore, changes in gland volume and tissue quality may hinder morcellation as well, increasing surgical times in this select group of patients $^{25,26}$. In our study, requiring a Foley at the time of HoLEP was not associated with increases in either enucleation or morcellation time. History of UTI, however, was associated with a modest increase in both surgical steps.

The impact of 5ARIs on prostatic tissue quality is not well understood but 5ARIs are known to affect the glandular to stromal ratio with an overall reduction in glandular tissue ${ }^{27-28}$. Therefore, hypothetically, long-term use of 5ARIs might increase the fibrous content of the prostate which could make enucleation and morcellation more difficult. We were surprised to find, therefore, that the use of 5ARI did not appear to affect our operative times. Sandfeldt et al reported a reduction in blood loss during transurethral resection of the prostate after 3 months of 
preoperative treatment with finasteride. However, in our experience, 5ARI use was not associated with any bleeding reduction during HoLEP ${ }^{29}$. Warner et al previously examined the impact of 5ARIs on HoLEP and reported that history of use does not impact HoLEP outcomes or operative time which is consistent with our findings ${ }^{30}$. Interestingly, in the current study we report that history of 5ARI was actually associated with a faster enucleation rate, although it did not reach statistical significance. Additionally, we observed shorter morcellation times in these patients. Whether these findings are related to random occurrence, reduced prostate volume associated with 5ARI use, or more senior participation during surgery remains unknown. In any event, there does not appear to be a clear relationship between 5ARI use and overall operative times during HoLEP.

This study is not devoid of limitations. It is retrospective in nature, although the data are collected in a prospective fashion. Additionally, complete preoperative data was not available for all patients and may have impacted results. As this study spans over a 15 year period, differences in patients and techniques may exist. Despite these limitations, this study is the first to examine how preoperative variables may impact enucleation and morcellation times in patients undergoing HoLEP for BPH.

\section{Conclusions}

HoLEP is an effective therapy for the management of BPH symptoms, particularly in men with large prostate glands. The duration of HoLEP is dependent upon prostate size, tissue quality, and surgeon's experience. A history of UTI is associated with an increase in operative time while anticoagulation is associated with a decrease in operative time. As surgeons gain proficiency and experience in performing HoLEP, enucleation and morcellation times decrease. 


\section{References}

1. Gilling PJ, Kennett K, Das AK, et al.: Holmium laser enucleation of the prostate (HoLEP) combined with transurethral tissue morcellation: an update on the early clinical experience. J Endourol 1998; 12: 457-459.

2. Tan AH, Gilling PJ, Kennett KM, et al.: A randomized trial comparing holmium laser enucleation of the prostate with transurethral resection of the prostate for the treatment of bladder outlet obstruction secondary to benign prostatic hyperplasia in large glands (40 to 200 grams). J Urol 2003; 170: 1270-1274.

3. Tan A, Liao C, Mo Z, et al.: Meta-analysis of holmium laser enucleation versus transurethral resection of the prostate for symptomatic prostatic obstruction. Br J Surg 2007; 94: 1201-1208.

4. McVary KT, Roehrborn CG, Avins AL, et al.: Update on AUA guideline on the management of benign prostatic hyperplasia. J Urol 2011; 185: 1793-1803.

5. Kuntz RM, Ahyal S, Lehrich K, et al. Transurethral holmium laser enucleation of the prostate versus transurethral electrocautery resection of theprostate: a randomized prospective trial in 200 patients. J Urol 2004; 172:1012-1016.

6. Salonia A, Suardi N, Naspro R, et al.: Holmium laser enucleation versus open prostatectomy for benign prostatic hyperplasia: an inpatient cost analysis. Urology 2006; 68: 302-306.

7. Kuo RL, Paterson RF, Kim SC, et al.: Holmium Laser Enucleation of the Prostate (HoLEP): A Technical Update. World J Surg Oncol 2003; 1: 6.

8. Krambeck AE, Handa SE and Lingeman JE: Experience with more than 1,000 holmium laser prostate enucleations for benign prostatic hyperplasia. J Urol 2010; 183: 1105-1109.

9. Elmansy HM, Kotb A and Elhilali MM: Holmium laser enucleation of the prostate: longterm durability of clinical outcomes and complication rates during 10 years of followup. $\mathrm{J}$ Urol 2011; 186: 1972-1976.

10. Wilson LC, Gilling PJ, Williams A, et al.: A randomised trial comparing holmium laser enucleation versus transurethral resection in the treatment of prostates larger than 40 grams: results at 2 years. Eur Urol 2006; 50: 569-573.

11. Kuntz RM, Ahyai S, Lehrich K, et al.: Transurethral holmium laser enucleation of the prostate versus transurethral electrocautery resection of the prostate: a randomized prospective trial in 200 patients. J Urol 2004; 172: 1012-1016.

12. Ahyai SA, Gilling P, Kaplan SA, et al.: Meta-analysis of functional outcomes and complications following transurethral procedures for lower urinary tract symptoms resulting from benign prostatic enlargement. Eur Urol 2010; 58: 384-397.

13. Naspro R, Bachmann A, Gilling P, et al.: A review of the recent evidence (2006-2008) for 532-nm photoselective laser vaporisation and holmium laser enucleation of the prostate. Eur Urol 2009; 55: 1345-1357.

14. Tinmouth WW, Habib E, Kim SC, et al.: Change in serum prostate specific antigen concentration after holmium laser enucleation of the prostate: a marker for completeness of adenoma resection? J Endourol 2005; 19: 550-554.

15. Humphreys MR, Miller NL, Handa SE, et al.: Holmium laser enucleation of the prostate-outcomes independent of prostate size? J Urol 2008; 180: 2431-2435; discussion 2435.

16. Krambeck AE, Handa SE and Lingeman JE: Holmium laser enucleation of the prostate for prostates larger than 175 grams. J Endourol 2010; 24: 433-437. 
17. Kim SC, Matlaga BR, Kuo RL, et al.: Holmium laser enucleation of the prostate: a comparison of efficiency measures at two institutions. J Endourol 2005; 19: 555-558.

18. Krambeck AE, Handa SE and Lingeman JE: Holmium laser enucleation of the prostate for prostates larger than 175 grams. J Endourol 2010; 24: 433-437.

19. Matlaga BR, Kim SC, Kuo RL, et al.: Holmium laser enucleation of the prostate for prostates of >125 mL. BJU international 2006; 97: 81-84.

20. Shah HN, Sodha HS, Kharodawala SJ, et al.: Influence of prostate size on the outcome of holmium laser enucleation of the prostate. BJU Int 2008; 101: 1536-1541.

21. Elzayat E, Habib E and Elhilali M: Holmium laser enucleation of the prostate in patients on anticoagulant therapy or with bleeding disorders. J Urol 2006; 175: 1428-1432.

22. El-Hakim A and Elhilali MM: Holmium laser enucleation of the prostate can be taught: the first learning experience. BJU Int 2002; 90: 863-869.

23. Gilling PJ, Kennett KM and Fraundorfer MR: Holmium laser enucleation of the prostate for glands larger than $100 \mathrm{~g}$ : an endourologic alternative to open prostatectomy. J Endourol 2000; 14: 529-531.

24. Shah HN, Mahajan AP, Hegde SS, et al.: Peri-operative complications of holmium laser enucleation of the prostate: experience in the first 280 patients, and a review of literature. BJU Int 2007; 100: 94-101.

25. Gandaglia G, Briganti A, Gontero P, et al.: The role of chronic prostatic inflammation in the pathogenesis and progression of benign prostatic hyperplasia (BPH). BJU Int 2013; 112: 432-441.

26. Mishra VC, Allen DJ, Nicolaou C, et al.: Does intraprostatic inflammation have a role in the pathogenesis and progression of benign prostatic hyperplasia? BJU Int 2007; 100: 327-331.

27. Marks LS, Partin AW, Gormley GJ, et al.: Prostate tissue composition and response to finasteride in men with symptomatic benign prostatic hyperplasia. J Urol 1997; 157: 2171-2178.

28. Marks LS, Partin AW, Dorey FJ, et al.: Long-term effects of finasteride on prostate tissue composition. Urology 1999; 53: 574-580.

29. Sandfeldt L, Bailey DM, Hahn RG: Blood loss during transurethral resection of the prostate after 3 months of treatment with finasteride. Urology 2001; 58: 972-976.

30. Warner JN, Nunez RN, Tyson MD, et al.: A multiinstitutional study of the effects of medical therapy for lower urinary symptoms on the perioperative outcomes of holmium laser enucleation of the prostate. Urology 2011; 78: 1385-1390. 
Table 1. Characteristics of patients undergoing HoLEP

\begin{tabular}{|c|c|}
\hline & Patients (n\%) \\
\hline Total patients & 960 \\
\hline Age (years), mean (SD) & $70.4(8)$ \\
\hline History of 5ARI use & $323(33.7)$ \\
\hline History of alpha blocker use & $696(72.5)$ \\
\hline Requiring Foley at time of HoLEP & $250(26.0)$ \\
\hline History of UTI & $393(40.9)$ \\
\hline History of anticoagulation & $201(20.9)$ \\
\hline Pre-HoLEP PSA (ng/ml), mean (range) & $8.1(0.04-449.4)$ \\
\hline TRUS weight (g), mean (SD) ${ }^{\mathrm{a}}$ & $102.8(51)$ \\
\hline PSA density, mean (SD) ${ }^{b}$ & $0.10(0.38)$ \\
\hline Beach ball identified & $34(3.5)$ \\
\hline Enucleation time (min), average (range) & $65.7(11-245)$ \\
\hline Morcellation time (min), average (range) & $19.7(3-260)$ \\
\hline \multicolumn{2}{|l|}{ HoLEP specimen weight (g) } \\
\hline$<30 \mathrm{~g}$ & $147(15.3)$ \\
\hline $30-100 \mathrm{~g}$ & $499(52.0)$ \\
\hline $100-150 \mathrm{~g}$ & $218(22.7)$ \\
\hline$>150 \mathrm{~g}$ & $96(10.0)$ \\
\hline Enucleation rate (g/min), average (SD) & $1.38(0.9)$ \\
\hline Morcellation rate (g/min), average (SD) & $5.32(2.8)$ \\
\hline Presence of prostate cancer & $84(8.8)$ \\
\hline Less experienced surgeon & $107(11.2)$ \\
\hline
\end{tabular}

${ }^{a}$ TRUS weight unavailable for 103/960

${ }^{\mathrm{b}}$ PSA density unavailable for $125 / 960$ 
Table 2. Predictors of enucleation time from multiple linear regression model

\begin{tabular}{|l|c|c|c|}
\hline Characteristic & $\begin{array}{c}\text { Coefficient } \\
\text { (minutes) }\end{array}$ & $95 \%$ CI & $\mathrm{p}$-value \\
\hline Requiring Foley at time of HoLEP & -3.49 & $-7.98-1.00$ & 0.127 \\
\hline History of UTI & 2.83 & $-0.71-6.38$ & 0.117 \\
\hline History of anticoagulation & -4.93 & $-9.22--0.64$ & 0.024 \\
\hline HoLEP specimen weight & & & \\
\hline$<30 g$ & Reference & & \\
\hline $30-100 \mathrm{~g}$ & 17.86 & $12.85-22.87$ & $<0.001$ \\
\hline $100-150 \mathrm{~g}$ & 31.55 & $25.76-37.33$ & $<0.001$ \\
\hline$>150 \mathrm{~g}$ & 39.81 & $32.69-46.93$ & $<0.001$ \\
\hline Less experienced surgeon & 20.86 & $15.28-26.44$ & $<0.001$ \\
\hline Beach ball present & -7.94 & $-17.38-1.50$ & 0.099 \\
\hline Constant & 43.76 & $39.00-48.52$ & $<0.001$ \\
\hline
\end{tabular}

Interpretation of linear regression model: for a patient not requiring a Foley at time of HoLEP but with a history of UTI and anticoagulation, who had a HoLEP specimen weight of 125g, with HoLEP performed by an experienced surgeon, and no beach balls, the estimated enucleation time is 73.2 minutes $(0+2.83-$ $4.93+0+31.55+0+0+43.76=73.2)$. Negative coefficients represent decreases in estimated morcellation time. 
Table 3. Predictors of morcellation time from multiple linear regression model

\begin{tabular}{|l|c|c|c|}
\hline Characteristic & $\begin{array}{c}\text { Coefficient } \\
\text { (minutes) }\end{array}$ & $95 \%$ CI & p-value \\
\hline History of 5ARI & -1.28 & $-3.32-0.77$ & 0.220 \\
\hline History of UTI & 2.45 & $0.50-4.40$ & 0.014 \\
\hline HoLEP specimen weight & & & \\
\hline$<30 \mathrm{~g}$ & Reference & & \\
\hline $30-100 \mathrm{~g}$ & 8.84 & $6.07-11.60$ & $<0.001$ \\
\hline $100-150 \mathrm{~g}$ & 21.59 & $18.39-24.78$ & $<0.001$ \\
\hline$>150 \mathrm{~g}$ & 39.71 & $35.77-43.65$ & $<0.001$ \\
\hline Less experienced surgeon & 3.00 & $-0.08-6.08$ & 0.056 \\
\hline Beach ball present & 25.26 & $20.10-30.42$ & $<0.001$ \\
\hline Constant & 4.47 & $1.83-7.10$ & 0.001 \\
\hline
\end{tabular}

Interpretation of linear regression model: for a patient with a history of 5ARI use, no history of UTI, HoLEP specimen weight of 125g, with HoLEP performed by a less experienced surgeon, and no beach balls present, the estimated morcellation time is 24.8 minutes $(-1.28+0+21.59+3.0+0+4.47=24.8)$.

Negative coefficients represent decreases in estimated enucleation time. 